\title{
Pump-probe spectroscopy of interminiband relaxation and electron cooling in doped superlattices
}

\author{
D. Stehr, ${ }^{\text {a) }}$ S. Winnerl, and M. Helm \\ Institute of Ion Beam Physics and Materials Research, Forschungszentrum Rossendorf, P.O. Box 510119, \\ 01314 Dresden, Germany \\ T. Dekorsy \\ Fachbereich Physik, Universität Konstanz, 78457 Konstanz, Germany
}

T. Roch and G. Strasser

Institut für Festkörperelektronik, TU Wien, Floragasse 7, 1040 Wien, Austria

\begin{abstract}
The picosecond dynamics of electrons in a doped GaAs/AlGaAs superlattice have been investigated by pump-probe experiments using an infrared free-electron laser. We observe a fast bleaching of the interminiband absorption followed by thermalization and a slower cooling component. The latter can lead to a positive or negative transmission change, resulting from the temperature dependence of the linear absorption spectrum at the respective wavelength. We show that the superlattice in contrast to quantum wells provides a unique picosecond thermometer for the electron temperature based on the dependence of the absorption on the electron distribution function.
\end{abstract}

The relaxation and cooling behavior of electrons in semiconductors and their heterostructures are of crucial importance for most optoelectronic devices. ${ }^{1}$ As a prime example the intersubband relaxation dynamics in quantum wells have to be known and well understood for the design of quantum cascade lasers. ${ }^{2-4}$ Many such investigations have been performed over the past two decades in quantum structures based on different materials systems, and an impressive level of insight has been obtained. ${ }^{5}$ Yet semiconductor superlattices (SLs), while representing a key model system of an artificial semiconductor structure, have been much less investigated in this respect, although they provide the basis of SL cascade lasers ${ }^{6}$ or Bloch oscillators. ${ }^{7}$ Compagnone et al. ${ }^{8}$ have calculated the interminiband relaxation rates and the stationary electron distribution function using a Monte Carlo technique, but no time resolved experiments have been performed to the best of our knowledge.

In this letter we present pump-probe transmission measurements on a doped $\mathrm{GaAs} / \mathrm{Al}_{0.3} \mathrm{Ga}_{0.7} \mathrm{As}$ superlattice using the infrared free-electron laser FELBE at the Forschungszentrum Rossendorf. ${ }^{9}$ We observe transient bleaching followed by interminiband relaxation on a picosecond time scale as well as heating and subsequent cooling of the electron system.

The superlattice was grown by molecular beam epitaxy (MBE) on a semi-insulating GaAs substrate. It consists of 300 periods of $9.0 \mathrm{~nm}$ thick quantum wells and $2.5 \mathrm{~nm}$ thick barriers, resulting in a width of 12 and $50 \mathrm{meV}$ for the first and second minibands, respectively. The wells are doped in the central $6 \mathrm{~nm}$ to $n=3 \times 10^{16} \mathrm{~cm}^{-3}$, corresponding to a density of $1.5 \times 10^{16} \mathrm{~cm}^{-3}$ averaged over one SL period and an areal density of $2 \times 10^{10} \mathrm{~cm}^{-2}$ per period. The Fermi energy at low temperature lies $1 \mathrm{meV}$ below the first miniband, when the impurity states are fully taken into account (see Ref. 10; neglecting impurity states it would lie $2.5 \mathrm{meV}$

${ }^{\text {a)} E l e c t r o n i c ~ m a i l: ~ d . s t e h r @ f z-r o s s e n d o r f . d e ~}$ above the bottom miniband edge). The linear interminiband absorption spectrum, measured on a sample with $45^{\circ}$ polished facets and one total internal reflection, is shown in Fig. 1 for different temperatures. At high temperature, the spectra reflect the van Hove singularities of the joint density of states at the center and edge of the mini-Brillouin zone, at low temperature they are dominated by impurity transitions (see inset of Fig. 1). ${ }^{10}$ We would like to point out that the strong temperature dependence of the absorption spectrum provides a means for measuring the effective temperature of the electron system. ${ }^{11}$

For the time-resolved experiments the above sample was placed into a LHe flow cryostat equipped with KRS-5 windows and excited with the free-electron laser (FEL), which provided bandwidth limited Gaussian pulses ${ }^{12}$ with a full width at half maximum (FWHM) between 1 and 2.5 ps (depending on the wavelength) at a $13 \mathrm{MHz}$ repetition rate. This

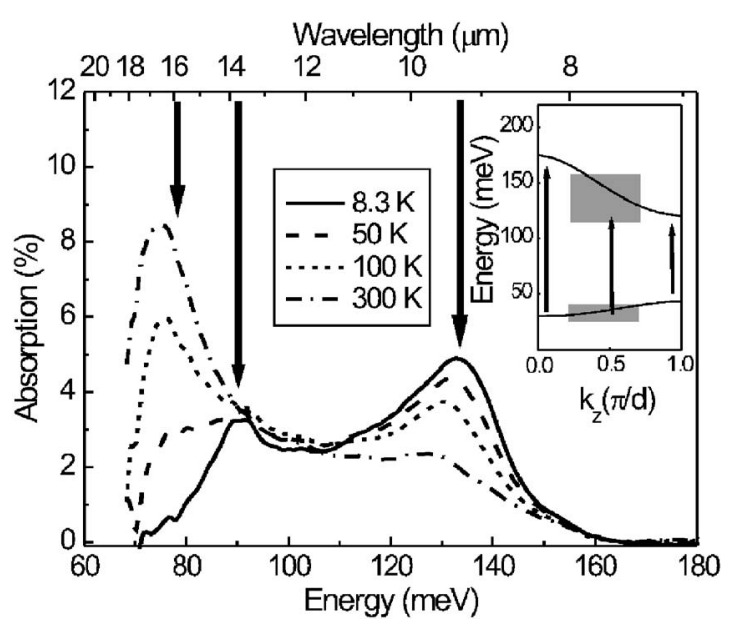

FIG. 1. Experimental infrared absorption spectrum of the sample at different temperatures. The arrows indicate the excitation wavelengths. The cutoff at $70 \mathrm{meV}$ is due to the detector employed in the measurement. The inset illustrates the interminiband and impurity transitions. 

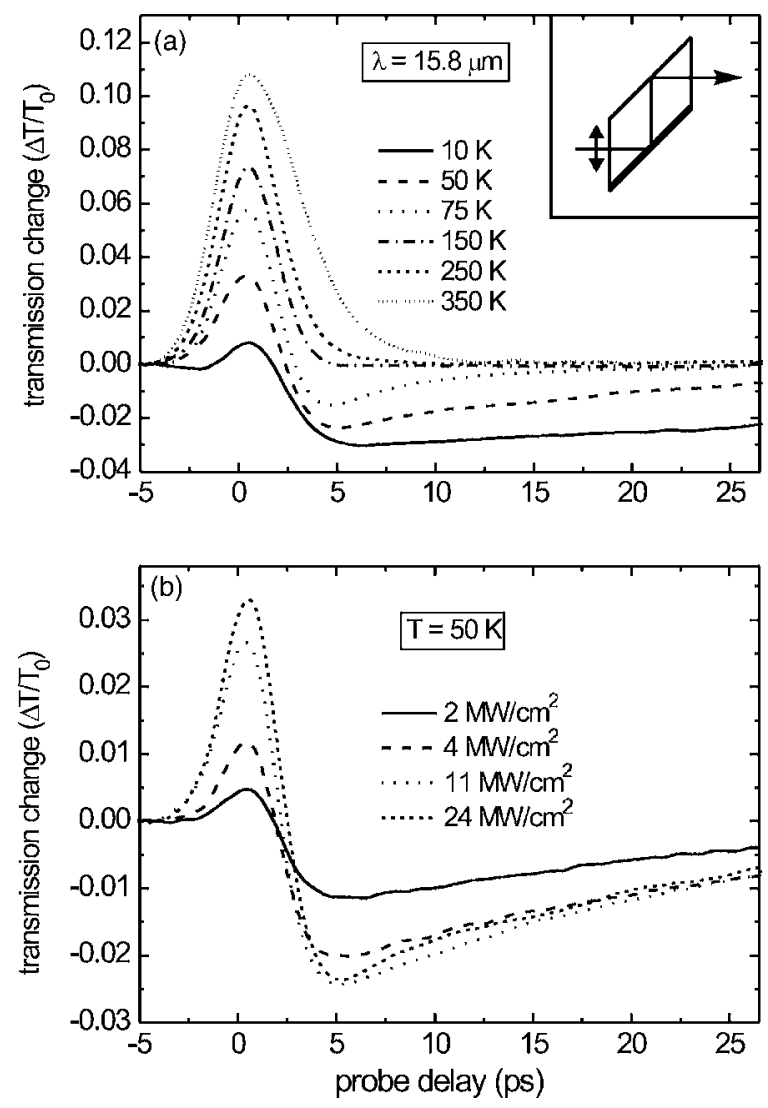

FIG. 2. Probe transmission as a function of pump-probe delay recorded at $15.8 \mu \mathrm{m}$ for different temperatures (a) and different pump intensities at $T=50 \mathrm{~K}(\mathrm{~b})$. Note that the intensity values are only accurate up to a factor of 2 approximately, while their relative values are accurate within $10 \%$. The inset illustrates the sample geometry.

high repetition rate results in a far superior signal-to-noise ratio compared to macropulsed FELs or kilohertz optical parametric amplifier systems. The maximum pulse energy used for excitation was $50 \mathrm{~nJ}$, corresponding to $650 \mathrm{~mW}$ average power, taking the sample geometry and attenuation of the cryostat windows into account. Under these conditions the lattice temperature of the samples increases by a few Kelvins. A fraction of the laser beam was split off using a Mylar beam splitter and served as the probe beam. Both pump and probe were focused onto the sample using a $10 \mathrm{~cm}$ focal-length off-axis parabolic mirror, taking care that the focal spot of the pump was larger than that of the probe. Behind the sample the transmitted probe beam was detected with a $\mathrm{LN}_{2}$ cooled mercury-cadmium telluride (MCT) detector. The signals were recorded by chopping the pump beam mechanically at $350 \mathrm{~Hz}$ and measuring the probe signal using a lock-in amplifier at this frequency. The pump-probe delay was achieved using a mechanical delay line.

Let us first analyze the pump-probe signals recorded at a wavelength of $15.8 \mu \mathrm{m}$ shown in Fig. 2. The FEL spectrum exhibited a FWHM of $\Delta \lambda=0.16 \mu \mathrm{m}$, which corresponds to a pulse duration of $2.2 \mathrm{ps}$ for bandwidth limited Gaussian pulses. By varying the lattice temperature [Fig. 2(a)] and the pump power [Fig. 2(b)] we observe drastic changes of the transient behavior. At low temperatures the induced transmission first rises due to bleaching of the transition, then decreases again within a few picoseconds and even goes negative, corresponding to induced absorption. This latter component relaxes on a much longer time scale. The fast bleaching component recovers by interminiband relaxation as well as by thermalization due to electron-electron scattering, which leads to a carrier distribution thermalized at a temperature higher than the lattice temperature. Exactly this is the reason for the negative (i.e., induced absorption) signal: from the linear absorption spectrum in Fig. 1 we know that at a wavelength of $15.8 \mu \mathrm{m}$ the absorption rises significantly with temperature. Thus an increase of the electron temperature leads to an increased absorption at this wavelength. The subsequent slower decay corresponds to the cooling of the electron distribution down to the lattice temperature. At higher temperature it is observed that $(i)$ the bleaching component becomes stronger, which is simply due to the fact that the linear absorption is larger, and (ii) the thermalization/cooling component becomes weaker and faster. This is because at higher temperature the opticalphonon related energy loss rate of the electrons increases, ${ }^{11}$ but also there is more energy absorbed according to the spectra in Fig. 1, leading to an even stronger energy loss. On the other hand the amplitude of the signal gets weaker, because at higher temperature the linear absorption does not change so much anymore (i.e., the intrinsic "thermometer" becomes less sensitive). The cooling time changes from $50 \pm 5$ ps at $T=10 \mathrm{~K}$ to $6 \pm 1 \mathrm{ps}$ at $T=75 \mathrm{~K}$. At $250 \mathrm{~K}$ the fast relaxation and slower cooling component nearly cancel each other, and at $T=350 \mathrm{~K}$ a single exponential interminiband relaxation of $2.2 \pm 0.1 \mathrm{ps}$ is observed.

The observations in Fig. 2(b), which show the intensity dependence of the signal at a constant lattice temperature of $50 \mathrm{~K}$, are consistent with the above picture: at higher pump intensity both the positive bleaching and the negative thermalization/cooling signal get larger and the cooling speeds up due to the larger temperature increase. Note that the vertical scale in Fig. 2 is quantitative: the bleaching at the highest pump power reaches $3 \%$, which corresponds to full saturation when compared with the vertical scale of the linear absorption spectrum (at $50 \mathrm{~K}$ and $15.8 \mu \mathrm{m}$ ). The negative heating signal reaches $2.4 \%$, which corresponds to a rise from $50 \mathrm{~K}$ to approximately $100 \mathrm{~K}$ when again compared to Fig. 1. Fitting an exponential decay on the cooling curve (not shown) yields cooling times from $40 \pm 4$ ps for the lowest to $18 \pm 2$ ps for the highest pump intensity.

We performed similar pump-probe measurements at two other wavelengths: one at the high-energy peak of the absorption spectrum at $\lambda=9 \mu \mathrm{m}$, which corresponds to transitions near the center of the mini-Brillouin zone, and at an intermediate wavelength of $13.75 \mu \mathrm{m}$. The used pump intensities were $40 \mathrm{MW} / \mathrm{cm}^{2}$ at $9 \mu \mathrm{m}$ and $20 \mathrm{MW} / \mathrm{cm}^{2}$ at $13.75 \mu \mathrm{m}$, respectively. From the laser spectrum we could estimate the pulselength to $1.5 \mathrm{ps}$ for $9 \mu \mathrm{m}$. These data are shown in Fig. 3. We clearly see the difference of the recorded signals: with shortening the wavelength, the induced absorption (at $15.8 \mu \mathrm{m}$ ) first becomes much smaller and finally turns into induced transmission in the case $\lambda=9.0 \mu \mathrm{m}$. However, this behavior is again completely consistent with the temperature dependence of the linear absorption spectra (Fig. 1). At $13.75 \mu \mathrm{m}$, the temperature change of the absorption is very small, e.g., we see a very small negative (i.e., induced absorption) signal (in other words, the intrinsic thermometer is not sensitive at this wavelength). At $\lambda=9.0 \mu \mathrm{m}$, it is obvious from Fig. 1 that the absorption decreases at higher temperatures. As a consequence now also the slow cooling transient is completely positive at all times. Due to the sim- 


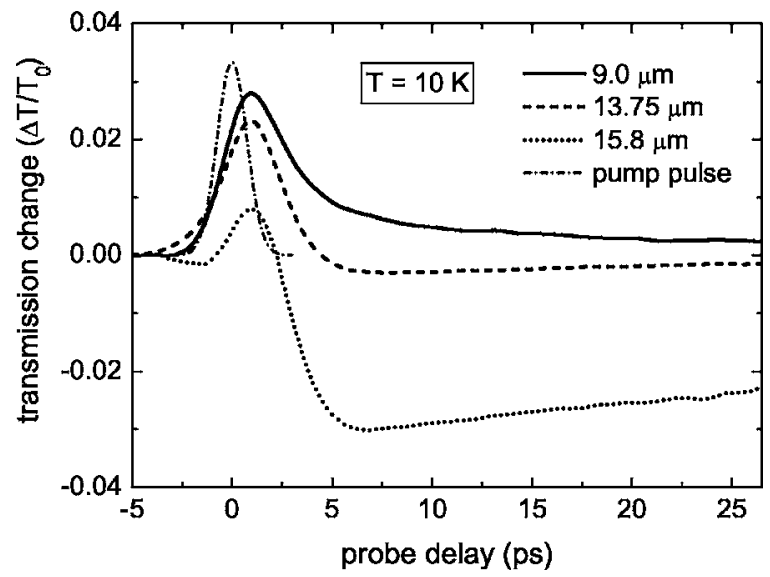

FIG. 3. Probe transmission as a function of pump-probe delay recorded at $T=10 \mathrm{~K}$ for different wavelengths as indicated. Also shown is the temporal pulse shape used to extract the decay times.

pler shape of the decay curve, it can now be fitted using a biexponential decay, yielding $\tau_{1} \approx 2.2 \pm 0.1 \mathrm{ps}$ and $\tau_{2} \approx 23 \pm 4 \mathrm{ps}$ as the best fit. While $\tau_{2}$ corresponds to the cooling time, $\tau_{1}$ reflects a combination of the interminiband relaxation and thermalization times of the excited electrons. The value agrees reasonably well with calculations for optical-phonon induced interminiband relaxation ${ }^{8}$ (these calculations were performed for InGaAs/InAlAs), indicating that electron-electron scattering cannot be much faster.

Due to the large spectral spreading of the interminiband absorption in a superlattice and its temperature dependence it is thus possible to clearly separate different contributions of the electron dynamics. In isolated quantum wells this is not possible due to the parallel dispersion of the subbands, or at least to a much lesser degree: Lutgen et al. ${ }^{13}$ were actually able to observe similar though much weaker effects in quantum wells due to the finite nonparabolicity of the subbands.

A similar behavior-positive and negative pump-probe signals-has also been observed by Woerner et al. ${ }^{14}$ for the inter-valence-band absorption in $p$-Ge. However, they ascribed both signal components to a change of carrier temperature (possible due to a nonmonotonic dependence of the absorption on the carrier temperature and a very fast holehole scattering) and excluded a real bleaching of the transition. Since in the present case the absorption depends monotonically on temperature for each wavelength, the fast signal must result from saturating the transition.
In summary, we have performed pump-probe measurements on interminiband transitions in GaAs/AlGaAs superlattices. We have observed a fast bleaching with subsequent relaxation and thermalization as well as a slower signal due to cooling of the hot electron distribution. The latter signal can be positive or negative, depending on the temperature dependence of the linear absorption at the respective wavelength, thus providing an internal thermometer for the electrons.

The authors are grateful to P. Michel and the whole ELBE team for their dedicated support, to W. Seidel for friendly collaboration, and to $\mathrm{H}$. Schneider for critical discussions.

${ }^{1}$ J. Shah, Ultrafast Spectroscopy of Semiconductors and Semiconductor Nanostructures (Springer, Berlin, 1999).

${ }^{2}$ C. Gmachl, F. Capasso, D. L. Sivco, and A. Y. Cho, Rep. Prog. Phys. 64, 1533 (2001).

${ }^{3}$ M. Troccoli, G. Scamarcio, V. Spagnolo, A. Tredicucci, C. Gmachl, F. Capasso, D. L. Sivco, A. Y. Cho, and M. Striccoli, Appl. Phys. Lett. 77, 1088 (2000)

${ }^{4}$ M. S. Vitiello, G. Scamarcio, V. Spagnolo, B. S. Williams, S. Kumar, Q. Hu, and J. L. Reno, Appl. Phys. Lett. 86, 111115 (2005).

${ }^{5}$ T. Elsaesser and M. Woerner, Phys. Rep. 321, 253 (1999).

${ }^{6}$ G. Scamarcio, F. Capasso, C. Sirtori, J. Faist, A. L. Hutchinson, D. L. Sivco, and A. Y. Cho, Science 276, 773 (1997).

${ }^{7}$ N. Sekine and K. Hirakawa, Phys. Rev. Lett. 94, 057408 (2005); P. G. Savvidis, B. Kolasa, G. Lee, and S. J. Allen, Phys. Rev. Lett. 92, 196802 (2004).

${ }^{8}$ F. Compagnone, A. DiCarlo, and P. Lugli, Appl. Phys. Lett. 80, 920 (2002); S. Tortora, F. Compagnone, A. DiCarlo, and P. Lugli, Physica E (Amsterdam) 7, 20 (2000).

${ }^{9}$ P. Michel, F. Gabriel, E. Grosse, P. Evtushenko, T. Dekorsy, M. Krenz, M. Helm, U. Lehnert, W. Seidel, R. Wünsch, D. Wohlfarth, and A. Wolf, Proceedings of the 26th International FEL Conference, Trieste, 2004. http://accelconf.web.cern.ch/AccelConf/f04/papers/MOAIS04/ MOAIS04.pdf

${ }^{10}$ D. Stehr, C. Metzner, M. Helm, T. Roch, and G. Strasser, Phys. Rev. Lett. 95, 257401 (2005). The impurity nature of the transition at low temperature is not expected to change the dynamics observed in this experiment, since the excited impurity band largely overlaps with the excited miniband.

${ }^{11}$ W. Hilber, M. Helm, K. Alavi, and R. Pathak, Appl. Phys. Lett. 69, 2528 (1996).

${ }^{12}$ U. Lehnert, P. Michel, W. Seidel, D. Stehr, J. Teichert, D. Wohlfarth, and R. Wünsch, Proceedings of the 27th International FEL Conference, Stanford, 2005. http://accelconf.web.cern.ch/AccelConf/f05/ papers/TUPP030.pdf

${ }^{13}$ S. Lutgen, R. A. Kaindl, M. Woerner, T. Elsaesser, A. Hase, and H. Künzel, Phys. Rev. B 54, R17343 (1996).

${ }^{14}$ M. Woerner, T. Elsaesser, and W. Kaiser, Phys. Rev. B 41, 5463 (1990). 